\title{
Extracranial Head and Neck, Peripheral Nerve Tumors: A 10-Year Experience at NCI, Egypt
}

\author{
Hala Aziz Shokralla1, Ahmed Elsayed Fathalla \\ ${ }^{1}$ Medical Oncology Department, National Cancer Institute, Cairo University, Cairo, Egypt \\ ${ }^{2}$ Surgical Oncology Department, National Cancer Institute, Cairo University, Cairo, Egypt \\ Email: halaaziz2001@gmail.com
}

How to cite this paper: Shokralla, H.A. and Fathalla, A.E. (2019) Extracranial Head and Neck, Peripheral Nerve Tumors: A 10Year Experience at NCI, EGYPT. Journal of Cancer Therapy, 10, 948-957. https://doi.org/10.4236/jct.2019.1012081

Received: November 4, 2019

Accepted: December 16, 2019

Published: December 19, 2019

Copyright $\odot 2019$ by author(s) and Scientific Research Publishing Inc. This work is licensed under the Creative Commons Attribution International License (CC BY 4.0).

http://creativecommons.org/licenses/by/4.0/ (c) (i) Open Access

\section{Abstract}

Background: Peripheral nerve tumors (PNT) originate from the neural crest and typically formed from Schwann cell sheath. Considering the number of nerves in this area, they form a very small percentage of neoplastic lesions of the head \& neck. A solitary mass in the lateral neck frequently confronts the surgeon. An uncommon cause for such a nodule is a neoplasm arising from nerve tissue with PNTs being the commonest of them to face. Aims: To study the clinico-pathological characteristics, presentations, surgical approaches, postoperative complications \& outcome of management. Materials \& $\mathrm{Me}-$ thods: a single institution combined retrospective \& prospective study. A retrospective analysis of all cases presented NCI-Cairo University with $\mathrm{H} \& \mathrm{~N}$ extracranial PNST candidate for surgery from Jan 2009 until the end of Dec 2015 \& prospective analysis of all cases with the same presentation presented to the NCI from Jan 2016 until the end of June 2019. 23 cases were included. Data were collected then analyzed. Results: Mean age was 34.7 years with female to male ratio 1.9:1. Mean size was $5.4 \mathrm{~cm}$. Commonest presentation was asymptomatic neck lump located laterally in the neck ( 9 cases, 39\%). CT scanning was done in 17 cases (74\%). As regard pathology, we have only 4 malignant cases (17\%). Most tumors (9 cases, 39\%) originated from cranial nerves. 18 cases $(78 \%)$ were resected through the neck. Complication occurred in 13 cases (56\%), most of them during tumor dissection from its nerve origin or root. Vascular complications occurred in 6 cases (26\%) due to bleeding controlled with ECA ligation (facial ischemia, hemiparesis \& paresthesia). Cranial nerve injury (XI, XII) occurred in two cases $(8.7 \%) \&$ Speech defects in other two 2 cases (8.7\%) due to maxillary resection. Postoperative radiation was given to all 4 malignant cases (17\%) of MPNST with Postoperative Chemotherapy to all cases experienced relapse after adjuvant RT. Post-operative palliative RT was given to ( 2 cases, $8.7 \%$ ) with bone deposits. Conclusion: The 
vast majority of $\mathrm{H} \& \mathrm{~N}$ extracranial PNTs are benign. Proper diagnosis is mandatory for ideal treatment. Complete R0 resection may not always be justified, therefore intracapsular or debulking procedures may have a role. The Malignant counterpart of PNTs (MPNST) is highly aggressive. They are treated with multimodality approach involving surgery with CTH \& RT.

\section{Keywords}

Peripheral Nerve Tumors, Outcome, NCI, Cairo University

\section{Introduction}

Embryologically, Peripheral nerve tumors (PNTs) originate from the neural crest and are typically formed from Schwann cell sheath. These cells of the sheath originate from a specialized population of neuro-mesenchymal cells, giving rise to the commonest subtypes; schwannomas and neurofibromas. Therefore, they may be named as peripheral nerve sheath tumors instead (PNSTs). These common two entities can arise from any cranial nerve, any spinal root with a sheath, any motor or sensory nerve, except I and II cranial nerves as they harbor no Schwann cell sheath \& are considered direct extension of the CNS [1].

As a group, head \& neck ( $\mathrm{H} \& \mathrm{~N})$ PNTs occur in $25 \%-45 \%$ of cases. They are reported most commonly in the parapharyngeal and retropharyngeal space, posterior pharyngeal wall, paranasal sinuses, nasal cavity, scalp, submandibular region, larynx, epiglottis, tongue, infratemporal fossa, oral cavity [2].

They comprise two categories with either benign or malignant behavior. The benign category includes: neurofibroma, schwannoma, perineuroma, cellular and mixed-type neurothecoma, nerve sheath myxoma and granular cell tumors. Neurofibroma \& schwannoma are the commonest; they compromise about $40 \%$ of cases. Benign non-neoplastic nerve tumors (Traumatic neuromas) can also occur. They are best called reactive, occurring after any nerve injury causing interrupted axons (neurotmesis). Regenerating axons lack endoneural tube to follow, which results in a mass of disorganized axons that is painful and tender [3] [4].

Malignant peripheral nerve sheath tumors (MPNSTs) are uncommon but are devastating tumors of peripheral nerve, representing only $10 \%$ of PNSTs. Their incidence in the general population is only $0.001 \%$. They are classified as malignant soft tissue sarcomas and can arise from a pre-existing plexiform neurofibromas or perineuriomas, or from normal nerves. They do not arise from schwannomas. $10 \%$ MPNSTs can occur as secondary neoplasms 10 to 20 years after radiation therapy. They may also appear in $22 \%$ to $50 \%$ of patients with neurofibromatosis type 1 (NF1), the rest being sporadic [5] [6].

The diagnosis of malignant versus benign tumor always requires a biopsy. Conventional imaging (CT \& MRI) may help but cannot reliably differentiate between malignant and benign lesions. CT scan is not the best imaging method 
as many tumors have signal densities similar to muscle and so are not well defined. However, CT can be useful in demonstrating bony remodeling adjacent to tumor, such as spinal neural foramina [7].

MRI is the most helpful imaging modality, especially in denoting the presence of a mass, determining whether the mass is intrinsic or extrinsic to a nerve, and delineating involvement of adjacent structures. MRI is less helpful in determining the pathology of a tumor as there are no pathognomonic signal characteristics for any type of tumor, but sometimes helpful in determining the malignant nature of a tumor. Rapid expansion of a known tumor, large size $(>5 \mathrm{~cm})$, heterogenicity, areas of hemorrhage \& necrosis, ill-defined margins, invasion of fat planes, and surrounding edema may suggest a MPNST. Still, a biopsy is needed for a definitive diagnosis. PET imaging can be helpful in distinguishing an MPNST from the benign neurofibroma counterpart and may be also important as part of a metastatic evaluation [8].

By gross inspection, MPNSTs tend to be large, firm tumors, containing areas of necrosis and hemorrhage. Microscopically, most MPNSTs are highly cellular, comprised of spindle cells remnants of Schwann cells. Variations include malignant triton tumor (MPNST with rhabdo-myosarcomatous differentiation), a highly malignant tumor containing embryonic striated muscle components [9] [10].

MPNSTs are staged and treated as malignant soft tissue sarcomas. However, wide excision in the H\&N region may be impossible due to the constraints of the local anatomy. Management of locally advanced or locally recurrent tumors is challenging. Initial radiation (RT) or combined chemoradiotherapy (CTH/RT) may permit some initially unresectable tumors to be easily resected. Even with aggressive surgical and combined CTH/RT treatment, the prognosis is disappointing. Poor prognostic signs include tumors $>5 \mathrm{~cm}$, higher grade, association with neurofibromatosis type 1 (NF1), older age, stage IV at diagnosis, and inability to achieve surgically gross tumor free margins (R0 resection) [9] [10] [11].

PNTs often present as an asymptomatic lateral neck mass. Sometimes, they can cause obstructive symptoms as nasal obstruction, dysphagia \& dysphonia, depending on their location and size. They may be associated with other diseases, such as multiple endocrine neoplasia (MEN) or neurofibromatosis type I. Pain will occur in the region of the tumor and any nerve the tumor involves, but pain may not be specific enough to discern the particular involved nerve. Neurologic deficits of sensory and motor function correspond to the nerve in which the tumor originates or which it is compressing, and will often be most useful in localizing the tumor. A Tinel sign over a nerve or tumor may also assist in localization to a particular nerve [12].

Considering they are relatively rare, these tumors should be considered in preoperative differential diagnosis, as other primary neck tumors may present as an asymptomatic neck mass; moreover, their resection may require neural reconstruction, and the surgeon must be prepared for this possibility. A critical issue regarding the treatment is the proper diagnosis, which is based on clinical 
findings, namely, the onset of a long-standing cervical mass. However, these tumors can also be discovered incidentally on imaging studies. The determination of the nerve of origin is strategic, since this provides the possibility of an informed decision by the patient and the surgeon about any risk of post-treatment functional sequelae. Although cytology may be of assistance, in most cases the technique of aspiration puncture is inconclusive [13] [14].

This cohort aims to study the clinico-pathological features of extracranial H\&N PNSTs and to present their clinical features, work-up, surgical management \& treatment outcome.

\section{Materials and Methods}

This is a single institution combined retrospective \& prospective study. A retrospective analysis of all cases presented NCI-Cairo University with $\mathrm{H} \& \mathrm{~N}$ extracranial PNTs candidate for surgery from Jan 2009 until the end of Dec 2015 \& prospective analysis of all cases with the same presentation presented to the NCI from Jan 2016 until the end of June 2019. 23 cases were included in this cohort. Data was collected from patients archive at the statistical department. Data included demographic features (age and sex), tumor characters (type, grade, stage, primary or recurrent) and surgical parameters (post excisional loss of nerve function). Treatment received; CTH, RT or both and their outcome. Treatment failure patterns were classified into local recurrence and distant metastasis.

Ethical clearance for the conduction of this study was obtained from our institute ethical committee.

\section{Results}

This study included 23 cases with PNTs (benign \& malignant).

Patients' age ranges from 2 to 78 years while mean age is 34.7 years. Females predominated this study (15 cases, $65 \%$ ) with female to male ratio of 1.9:1. mean tumor size was $5.4 \mathrm{~cm}$ (ranged from 3 to $10 \mathrm{~cm}$ ).

The main presenting symptom was neck lump (11cases, $48 \%$ ); other patients' complains were recurrence of an old lesion (4 cases, 17\%) or nasal mass ( 3 cases, 13\%) (Table 1).

The commonest radiological investigation used was CT scanning (17 cases, $74 \%$ ), followed by CT angiography ( 9 cases, $39 \%$ ). Neck U/S (4 cases, $17 \%$ ), MRI ( 4 cases, $17 \%$ ), while no imaging study was requested in (6 cases, $26 \%$ ) specially in skin nodules and oral cavitary lesions (Table 2).

Most of tumors (11 cases, $48 \%$ ) originated from cranial nerves mainly VII, X, $\mathrm{XI}$, and XII. four cases (17\%) from the brachial plexus, three cases (13\%) from cervical plexus, 2 cases (9\%) from cervical sympathetic chain, 2 cases (9\%) C5 \& C6 roots; and in only one case (4\%) the anatomical neurological origin was not clear. The commonest benign tumor in this cohort was schwannoma (8 cases, $35 \%$ ) while MPNST was found only in 4 cases (17\%) (Table 3 ). 
Table 1. Different presentations in our cohort (23 cases, $100 \%)$.

\begin{tabular}{cc}
\hline Neck lump & $(11$ cases, $48 \%)$ \\
Recurrence of an old lesion & $(4$ cases, $17 \%)$ \\
Nasal mass & $(3$ cases, $13 \%)$ \\
Oral cavity lesion & $(2$ cases, $9 \%)$ \\
Skin nodules & $(2$ cases, $9 \%)$ \\
Otalgia \& headache & $(1$ case, $4 \%)$ \\
\hline
\end{tabular}

Table 2. Anatomic site of 23 Head and Neck PNTs \& Diagnostic modality used.

\begin{tabular}{ccccc}
\hline Location & $\boldsymbol{N}(\%)$ & Imaging & FNAC & Open Biopsy \\
\hline Lateral & $11(48 \%)$ & 8 & 2 & 1 \\
Supraclavicular & $6(26 \%)$ & 3 & 1 & 2 \\
Submandibular & $3(13 \%)$ & 2 & 1 & 0 \\
Parotid & $2(9 \%)$ & 1 & 0 & 1 \\
Frontoethmoidal & $1(4 \%)$ & 1 & 0 & 0 \\
\hline
\end{tabular}

FNAC: fine needle aspiration cytology.

Table 3. The mainhistologic pattern (23 cases, 100\%).

\begin{tabular}{cc}
\hline Schwannoma & $8(35 \%)$ \\
Neurofibroma & $6(26 \%)$ \\
Neurofibromatosis & $3(13 \%)$ \\
Granular cell tumor & $2(9 \%)$ \\
MPNST (neurofibrosarcoma) & $4(17 \%)$ \\
\hline
\end{tabular}

\section{Management}

Surgical management was the main line of treatment in our study conducted in all patients ( 23 cases). The majority of our patients (18 cases, $78 \%$ ) were attacked through the neck. only 2 patients (9\%) presented with maxillary fullness \& epistaxis underwent a trans-sinus biopsy through Caldwell-Luc procedure followed by total maxillectomy as a result of complete sinus destruction combined by reconstruction with obturator prothesis application. Intracapsular enucleation was done 3 cases (13\%) with a preoperative diagnosis of cervical neurofibroma.

2 cases (9\%) with neurofibroma presented with pathological bone erosions in C5 and C6 vertebral bodies as a result of pressure necrosis. These patients underwent resection of the mass with its origin from $\mathrm{C} 5$ root together with parts of the eroded C5 \& C6 vertebral body which required posterior spinal fixation with vertebral plates and screws.

Post-resection adjuvant radiotherapy (RT) was received in the 4 cases (17\%) of MPNST to decrease local recurrence.

Chemotherapy (CTH) was given in cases where relapse occurred in form of Adriamycin-ifosfamide regimen as for soft tissue sarcomas. Two cases relapse in 
form of lung metastasis (9\%) and two others cases (9\%) in lungs and bone. They received both $\mathrm{CTH}$ and palliative $\mathrm{RT}$ to bone.

Surgical complications had occurred in 13 cases (56\%), mostly during tumor dissection from its nerve origin or root resulting in neuropraxia in 11 cases (48\%). Permanent cranial nerve injury (XI, XII) in 2 cases (9\%). Vascular complications occurred in 6 cases (26\%) due to the need for intraoperative bleeding control through external carotid artery ligation resulting in (facial ischemia, hemiparesis \& paresthesia). Speech defect (nasal tone) \& water regurgitation from nose occurred in 2 cases (9\%) due to maxillary resection even after obturator prothesis insertion.

\section{Discussion}

Peripheral nerve tumors (PNTs) originate from the neural crest and are typically formed from Schwann cell sheath. Schwannomas has an exclusive origin from Schwann cells, while neurofibromas comprise a mixture of three cells origin: Schwann cells, perineural cells \& perineural fibroblasts [15].

This cohort included 23 patients with benign and malignant PNT arising in the head and neck region. The gross and microscopic characteristics of these tumors were related to their prognosis and treatment $\&$ paralleled those seen in other studies. The mean age of patients was 34.7 years ranged from 2 to 78 years, with predominance of females ( 15 females/ 8 males). The size of these tumors can range from a few millimeters to $25 \mathrm{~cm}$ in most studies. In this cohort, the mean size was $5.4 \mathrm{~cm}$ (ranged from 3 to $10 \mathrm{~cm}$ ).

Serhrouchni KI et al. reported that PNT can arise at any age with no age or race prevalence while other studies reported peculiar age prevalence to the fifth decade of life [16].

Benign PNTs mainly schwannomas are indolent slowly growing tumors, almost often are encapsulated tumor and isolated. They are always connected to the nerve of origin which sometimes cannot be identified clearly [17].

Neurofibromas can present as an isolated tumor or in a multipleform pattern. Localized neurofibromas usually arise from cutaneous nerves, with occasional involvement of deep nervous sheath. They are benign, slow-growing, circumscribed usually non-capsulated tumors. They may occur primarily as solitary neurofibromas or in about $10 \%$ of cases are associated with neurofibromatosis type I (a somatic mutation in the NF1gene, i.e.; a tumor suppressor gene located on chromosome 17) [18].

Schwannomas may show cystic degeneration \& areas of hemorrhagic necrosis. These changes are not seen in cases of neurofibromas. Nerve dysfunction may occur (vocal cord paralysis due to $\mathrm{X}$ affection, Horner syndrome due to cervical sympathetic chain involvement), UL sensory or motor dysfunction. In our study, the commonest symptomatology was neck lump (9 cases, 39\%), others included recurrence of an old lesion ( 4 cases, 17\%), nasal mass ( 3 cases, 13\%), oral cavity lesion ( 2 cases, $9 \%)$, skin nodules ( 2 cases, $9 \%)$ or headache $\&$ otalgia ( 1 cases, 
4\%) [17] [18].

The commonest anatomical site of origin of these tumors is the parapharyngeal region, usually retro-styloid in the carotid fossa causing anterior displacement of the fat present in the pre-styloid parapharyngeal space \& bulging of lateral wall of oropharynx. Other sites include submandibular region, paranasal sinuses, face \& oral cavity. In this cohort, most of cases presented as a lateral neck mass (9 cases, 39\%), supraclavicular region (6 cases, $26 \%$ ), submandibular space ( 3 cases, $13 \%$ ), parotid fossa ( 2 cases, $9 \%) \&$ frontoethmoidal sinus ( 1 case, 4\%) [19].

Diagnostic difficulties may arise sometimes during histopathology as features of the pseudo-epitheliomas hyperplastic lesions can be confused with the early well-differentiated oral cavity squamous cell carcinoma [20].

Regarding MPNST, Loree et al. reported that local recurrence correlated with tumor size and resection margin status. All cases received post operative RT to decrease rate local recurrence.

None of our cases experienced local recurrence but all the relapsed four cases (17\%) presented with systemic failure in lungs or bone for which they receive chemotherapy in form of (Adriamycin-ifosfamide) as for soft tissue sarcomas and palliative radiation for bone metastasis.

Tumor grade predict for development of distant metastases. Negative margins are crucial for obtaining local control with adjuvant radiotherapy that may be beneficial in this group of patients with such an aggressive tumor. Salvage surgery may be feasible for those patients with local recurrence [9] [20].

Although, imaging studies should clarify the nerve of origin which provides a clear decision by the patient for any functional sequelae following surgery, this is not usually achieved. Cytology may be of assistance; in most cases the technique of aspiration puncture is inconclusive. The specificity of imaging studies is also not high. These investigations are of benefit mainly evaluation of the vascularization of tumors, but may be less useful for diagnosis of the tumor's nerve of origin [7] [8] [21].

Among the differential diagnoses that should be encounterd, reactive or metastatic lymph node diseases from other H\&N malignancy; benign soft tissue tumors as (fibroma, leiomyoma, lipoma \& paraganglioma); carotid artery aneurysm; and branchial plexus malformations [4] [18].

The choice between resection of the tumor included with the nerve of origin or intracapsular enucleation with possibility of preserving nerve function is determined by the relationship between the tumor and the nerve of origin. Intracapsular enucleation is feasible in eccentrically located tumors, without dispersing the neural fibers, however, this is not the case with MPNST where complete $\mathrm{R} 0$ resection is the rule that may unfortunately result in permanent nerve morbidity [3] [5] [21] (Figures 1-3).

\section{Conclusion}

The vast majority of extracranial PNTs are benign. Proper diagnosis through a 

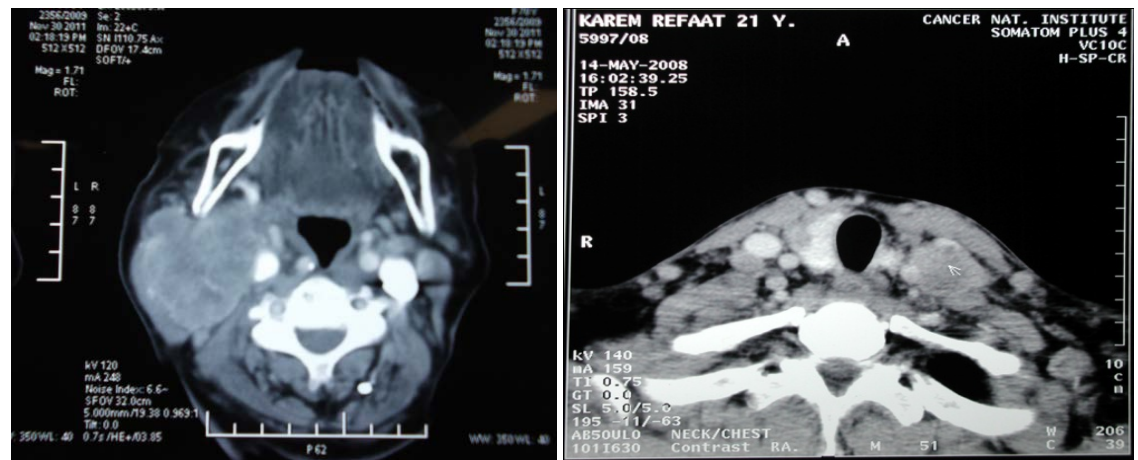

Figure 1. CT scan; axial views for 2 patients: the left with LT. lateral neck mass proved to be schwannoma \& the right one with Rt.neck lump proved to be neurofibroma.

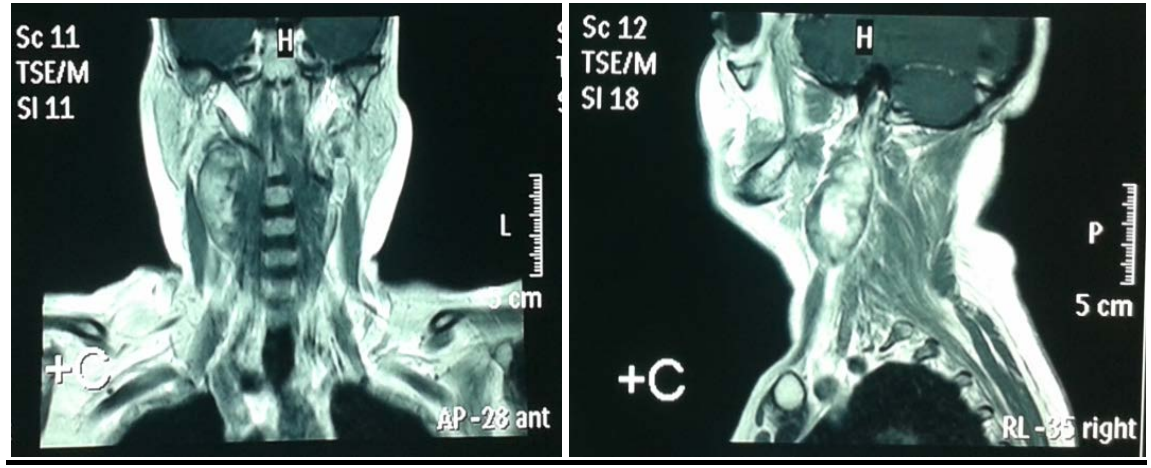

Figure 2. MRI (coronal \& sagittal views) showing parapharyngeal space mass proved to VII nerve schwannoma.

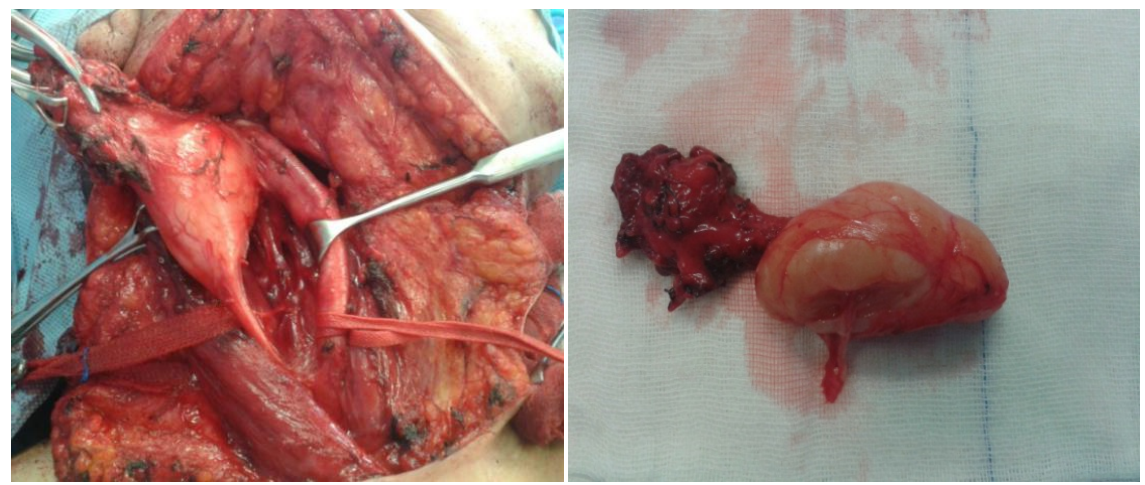

Figure 3. (left) intraoperative view of a case of vagal nerve schwannoma with the postoperative specimen (right).

high index of suspicion \& optimum preoperative evaluation. Ideal treatment involves complete R0 resection; however, debulking procedures may have a definite role. Malignant counterpart of the PNTs (MPNST) is aggressive and is treated with radical surgical resection followed by radiation therapy. Chemotherapy is reserved for metastatic disease.

\section{Conflicts of Interest}

The authors declare no conflicts of interest regarding the publication of this paper. 


\section{References}

[1] Barnes, L., Eveson, J.W., Reichart, P. and Sidransky, D. (2005) World Health Organization Classification of Tumours. Pathology and Genetics of Head and Neck Tumours. International Agency for Research on Cancer, Liyon.

[2] Shah, J.P., Patel, S.G. and Singh, B. (2012) Head and Neck Surgery and Oncology. Elsevier Health Sciences, Amsterdam.

[3] Curioni, O.A., et al. (2015) Extracranial Neurogenic Tumors of the Head and Neck. Brazilian Journal of Otorhinolaryngology, 81, 604-609. https://doi.org/10.1016/j.bjorl.2015.08.012

[4] Golan, J.D. and Jacques, L. (2004) Nonneoplastic Peripheral Nerve Tumors. Neurosurgery Clinics of North America, 15, 223-230. https://doi.org/10.1016/j.nec.2004.02.001

[5] Baehring, J.M., Betensky, R.A. and Batchelor, T.T. (2003) Malignant Peripheral Nerve Sheath Tumor: The Clinical Spectrum and Outcome of Treatment. Neurology, 61, 696-698. https://doi.org/10.1212/01.WNL.0000078813.05925.2C

[6] Suárez, C., Rodrigo, J.P., Ferlito, A., Cabanillas, R., Shaha, A.R. and Rinaldo, A. (2006) Tumours of Familial Origin in the Head and Neck. Oral Oncology, 42, 965-978. https://doi.org/10.1016/j.oraloncology.2006.03.002

[7] Pilavaki, M., Chourmouzi, D., Kiziridou, A., Skordalaki, A., Zarampoukas, T. and Drevelengas, A. (2004) Imaging of Peripheral Nerve Sheath Tumors with Pathologic Correlation: Pictorial Review. European Journal of Radiology, 52, 229-239. https://doi.org/10.1016/j.ejrad.2003.12.001

[8] Saito, D.M., Glastonbury, C.M., El-Sayed, I.H. and Eisele, D.W. (2007) Parapharyngeal Spaces Schwannomas: Preoperative Imaging Determination of the Nerve of Origin. Archives of Otolaryngology_Head and Neck Surgery, 133, 662-667. https://doi.org/10.1001/archotol.133.7.662

[9] Loree, T.R., North Jr., J.H., Werness, B.A., Nangia, R., Mullins, A.P. and Hicks Jr., W.L. (2000) Malignant Peripheral Nerve Sheath Tumors of the Head and Neck: Analysis of Prognostic Factors. Otolaryngology-Head and Neck Surgery, 122, 667-672. https://doi.org/10.1067/mhn.2000.106191

[10] Stasik, C.J. and Tawfik, O. (2006) Malignant Peripheral Nerve Sheath Tumor with Rhabdomyosarcomatous Differentiation (Malignant Triton Tumor). Archives of Pathology \& Laboratory Medicine, 130, 1878-1881.

[11] Bernier, J. (2011) Head and Neck Cancer: Multimodality Management. Springer Science \& Business Media, Berlin.

[12] Biswas, D., Marnane, C.N., Mal, R. and Baldwin, D. (2007) Extracranial Head and Neck Schwannomas: A 10-Year Review. Auris Nasus Larynx, 34, 353-359. https://doi.org/10.1016/j.anl.2007.01.006

[13] Sharma, D.K., Sohal, B.S., Parmar, T.L. and Arora, H. (2012) Schwannomas of Head and Neck and Review of Literature. Indian Journal of Otolaryngology and Head \& Neck Surgery, 64, 177-180. https://doi.org/10.1007/s12070-011-0248-0

[14] Dey, P., Mallik, M.K., Gupta, S.K. and Yasishta, R.K. (2004) Role of Fine Needle Aspiration Cytology in the Diagnosis of Soft Tissue Tumours and Tumour-Like Lesions. Cytopathology, 15, 32-37. https://doi.org/10.1046/j.0956-5507.2003.00102.x

[15] Bhattacharyya, A.K., Perrin, R. and Guha, A. (2004) Peripheral Nerve Tumors: Management Strategies and Molecular Insights. Journal of Neuro-Oncology, 69, 335-349. https://doi.org/10.1023/B:NEON.0000041891.39474.cb

[16] Serhrouchni, K.I., Chbani, L., Hammas, N., Kamal, D., El Fatemi, H., Harmouch, T., 
et al. (2014) Two Rare Schwannomas of Head and Neck. Diagnostic Pathology, 9, 27. https://doi.org/10.1186/1746-1596-9-27

[17] Sharma, D.K., Sohal, B.S., Parmar, T.L. and Arora, H. (2012) Schwannomas of Head and Neck and Review of Literature. Indian Journal of Otolaryngology and Head \& Neck Surgery, 64, 177-180. https://doi.org/10.1007/s12070-011-0248-0

[18] Kim, D.H., Murovic, J.A., Tiel, R.L., Moes, G. and Kline, D.G. (2005) A Series of 397 Peripheral Neural Sheath Tumors: 30-Year Experience at Louisiana State University Health Sciences Center. Journal of Neurosurgery, 102, 246-255. https://doi.org/10.3171/jns.2005.102.2.0246

[19] Skandalakis, L.J. and Skandalakis, J.E. (2013) Surgical Anatomy and Technique: A Pocket Manual. Springer Science \& Business Media, Berlin.

[20] Oliai, B.R., Sheth, S., Burroughs, F.H. and Ali, S.Z. (2005) "Parapharyngeal Space" Tumors: A Cytopathological Study of 24 Cases on Fine-Needle Aspiration. Diagnostic Cytopathology, 32, 11-15. https://doi.org/10.1002/dc.20154

[21] Levi, A.D., Ross, A.L., Cuartas, E., Qadir, R. and Temple, H.T. (2010) The Surgical Management of Symptomatic Peripheral Nerve Sheath Tumors. Neurosurgery, 66, 833-840. https://doi.org/10.1227/01.NEU.0000367636.91555.70 